\title{
Diagnostic value of thiols, paraoxonase 1 , arylesterase and oxidative balance in colorectal cancer in human
}

\author{
N. BULBULLER ${ }^{1}$, E. EREN ${ }^{2}$, H. Y. ELLIDAG ${ }^{3}$, O. Z. ONER ${ }^{1}$, C. SEZER ${ }^{4}$, O. AYDIN ${ }^{3}$, N. YILMAZ ${ }^{3, *}$ \\ ${ }^{1}$ General Surgery Clinic of Antalya Education and Research Hospital of Ministry of Health, Antalya, Turkey; ${ }^{2}$ Antalya Public Health Center of \\ Ministry of Health, Antalya, Turkey; ${ }^{3}$ Central Laboratories of Antalya Education and Research Hospital of Ministry of Health, Antalya, Turkey; \\ ${ }^{4}$ Pathology Laboratory of Antalya Education and Research Hospital of Ministry of Health, Antalya, Turkey
}

*Correspondence: necatyilmaz@hotmail.com

Received November 7, 2012 / Accepted January 7, 2013

\begin{abstract}
Oxidative stress has been shown to play an important role in carcinogenesis. We hypothesize that serum oxidative and anti-oxidative factors work together to influence colon and rectal cancer through an oxidative balance mechanism. Total thiol (Ttl) is considered a plasma antioxidant and high density lipoprotein (HDL)- dependent paraoxonase l (PON1) is known as a free radical scavenger. This study was undertaken to determine the activity of PON1, arylesterase (ARE) and Ttl levels and oxidative balance in colorectal cancer (CRC) patients and healthy subjects.

Paraoxonase and arylesterase activities of the HDL-dependent PON1 enzyme and the level of Ttl, total oxidant status (TOS) ,total antioxidant status (TAS) in forty patients with CRC (19 female, 21 male; mean age, $56.5 \pm 2.1)$ and thirty-nine age and sex matched healthy persons ( 22 female, 17 male; mean age $56 \pm 1.7$ ) were studied.

PON1 and ARE activities were significantly lower in patients with CRC compared to controls ( $p<0.001$ for each), whereas oxidant parameters (TOS and OSI) were significantly higher in CRC patients $(p<0.001$ for each). However, TAS and Ttl significantly decreased in patients with CRC ( $p=0.03$ and $\mathrm{p}<0.0001$, respectively). According to the ROC curve analysis, Ttl was superior to other parameters in terms of diagnostic sensitivity and specificity which were $97.5 \%$ and $92.3 \%$, respectively, and the AUC 0.97 in CRC patients.

Decreased Ttl and HDL-dependent PON 1 enzyme activities and high TOS levels imply an imbalance of the free radical system which enhances the support the hypothesis that CRC is associated with excess reactive oxygen species. These findings show that of serum oxidative imbalance was associated with an increased risk of CRC. To our knowledge, this is the first report demonstrating lower serum PON1, ARE activities in CRC patients. The mechanism behind this association needs further elucidation.
\end{abstract}

Key words: colon cancer, thiols, oxidative stress

Inflammation and oxidative stress contribute to the etiology of almost every known disease. Oxidative stress has long been associated with the pathophysiology of cancer. In particular, enhanced reactive oxygen species (ROS) formation increases DNA damage, genome instability, and cell proliferation especially during cancer initiation. On the other hand, oxidative stress also counteracts tumorigenesis, as it induces senescence and drives apoptosis and other cell death pathways. ROS generated by enzymatic and non enzymatic systems modify lipids and sterols, producing oxidized lipids and oxidized sterols that, if unchecked, produce a vicious cycle of undesirable inflammation and more oxidative stress. The precise spatiotemporal control of ROS generation is therefore a critical regulator of cell survival and death, for instance since overwhelming mitochondrial oxidative stress exerts apoptotic rather than protumorigenic functions. Nevertheless, ROS may be conducive to the vitality of cancer cells and drive signaling transduction pathways, which lead to activation of redox sensitive transcription factors and genes involved in cancer cell growth, proliferation, and survival [1].

The presence of an organophosphate hydrolysing enzyme was first demonstrated in mammalian tissue, an observation that ultimately led to the identification of a human paraoxonase in serum in 1953 [2]. Paraoxonase - so named because of its ability to hydrolyse the toxic metabolite of parathion, paraoxon - was also shown early after its identification to 
manifest arylesterase-lactonase activity [3]. The antioxidative effects of PON1, PON2 and PON3 were reported long ago, but underlying mechanisms were uncovered just recently. Most studies in the field of paraoxonases (PONs) deal with cardiovascular diseases, such as atherosclerosis and diabetes, where PONs exert protective functions in cell culture as well as animal studies. Based on the latest knowledge, these enzymes modulate execution of the apoptotic program [4].

Pro-oxidant and anti-oxidant genetic and lifestyle factors can contribute to an individual's level of oxidative stress [5]. Oxidative stress has been shown to play an important role in carcinogenesis, but prospective evidence for an association between biomarkers of oxidative stress and colorectal cancer (CRC) risk is limited [6]. PON1 levels and activity are lower in many inflammatory and oxidative stress-associated diseases [7]. Also, serum PON1 and arylesterase activities were reduced in patients with ovarian, breast, prostate and lung cancer [810]. These studies clearly demonstrate a link between PON1 and cancer etiology.

Previously it was demonstrated that the paraoxonase $(\mathrm{PON} 1 / 2 / 3)$ genes and proteins were expressed in human intestinal biopsies [11]. Also, PON1 mRNA was expressed in colon [12]. It is possible that HDL delivers PON1 to the tissues, as HDL is abundant in the tissue fluid. In many of the tissues studied, PON1 immunostaining was found within the cell where it could protect lipids within internal cell membranes against oxidative damage [13]. However, how the PON1 protein is translocated from HDL to internal structures within the cell is unknown [14].

Rothem L et al. [11] reported that significantly fewer biopsies expressed PON1 and PON3 in the duodenum of celiac patients, in the terminal ileum of Crohn's patients, and in the colon of ulcerative colitis patients compared to controls. However, the wide tissue distribution of PON1 protein would theoretically lead to greater protection against oxidative stress for these tissues.

Serum concentrations of different oxidant species can be measured separately in laboratories; but the measurements are time-consuming, too expensive, and require complicated techniques. Recently, lipid peroxidation levels were monitored by determining total oxidant status (TOS) [15]. Besides, total antioxidant status (TAS) is a useful estimate of the activity of antioxidants in a medium [16]. Therefore, measurements of TAS and TOS can provide information on an individual's overall serum oxidative stress index (OSI), which may include those antioxidants and oxidants not yet recognized or not easily measured [17].

Thiols ( $\mathrm{Ttl}$ ) are endogenous molecules that contain the sulfhydryl group (-SH) attached to a carbon atom. They support aerobic cells to maintain a reducing state, despite an oxidising environment [18]. These molecules are extremely efficient antioxidants, and they protect cells from the results of damage caused by free radicals, due to their ability to react with the latter [19]. Both intracellular and extracellular redox states of thiols play a critical role in the determination of protein structure and function, regulation of enzymatic activity of transcription factors and antioxidant protection [20].

According to our knowledge, oxidative/antioxidative balance, and particularly PON1 enzyme activities in colorectal cancer have never been studied. Therefore, we aimed to determine the diagnostic value of measuring new markers of oxidative stress in individuals of CRC.

\section{Material and methods}

Subjects. Forty patients (19 female, 21 male; mean age, $56.5 \pm 2.1$ ) who were presented at the Surgery Clinic of Education and Research Hospital were prospectively included in the study. Thirty-nine age- and sex-matched healthy control subjects ( 22 female, 17 male; mean age $56 \pm 1.7$ ) were also enrolled for comparison.

The patients (newly diagnosed-not medically treated) and control volunteers were asked to fulfill a questionnaire, and gave informed consent before starting the study. The following data were recorded for each patient: age, sex, physical activity, education, consumption of fruit, vegetables, meat, fish, fibre, chronic treatments, alcohol consumption, smoking, family history of gastrointestinal malignancy. All the participants belonged to the same ethnic group and had comparable lifestyle habits and socioeconomic status All subjects had full physical examination. Consequently, blood pressure was measured manually with a sphygmomanometer. Body mass index was calculated as weight in kilograms divided by height in meters squared.. The patients with CRC, or subjects in the control group with an unstable medical condition (e.g cardiovascular, hepatic, renal or endocrine disorder) within the past 2 years; or the ones using any medications - e.g. lipid lowering agents or antioxidant drugs - that could influence the results, were excluded. Final diagnosis of each patient was confirmed by the microscopic evaluation of colonoscopic biopsies, followed by total excision of tumors. The final pathology reports of patients were evaluated, and subjects were divided into 3 groups according to the tumor grades. This study was performed in accordance with the ethical standards set by the Declaration of Helsinki and was approved by the local ethics committee.

Analytical methods. Blood sample collection. Blood samples were obtained after an overnight fasting state. Serum samples were then separated from the cells by centrifugation at $3000 \mathrm{rpm}$ for 10 minutes, and portioned. The levels of triglycerides (TG), total cholesterol (TC), HDL-cholesterol (HDL-C) and LDL-cholesterol (LDL-C) were determined by using commercially available assay kits (Abbott) with an autoanalyzer (Architect ${ }^{\circ} \mathrm{c} 16000$, Abbott Diagnostics) and these parameters were studied immediately. Serum portions were stored at $-80^{\circ} \mathrm{C}$ and used to analyze PON1, ARE, Ttl, TOS and TAS.

Measurement of paraoxonase and arylesterase enzyme activities in serum. PON1 and ARE enzyme activities were 
measured by using commercially available kits (Relassay, Turkey). Fully automated PON1 activity measurement method consists of two different sequential reagents; the first reagent is an appropriate Tris buffer and it also contains calcium ion, which is a cofactor of PON1 enzyme. Linear increase of the absorbance of $p$-nitrophenol, produced from paraoxon, is followed at kinetic measurement mode. Nonenzymatic hydrolysis of paraoxon was substracted from the total rate of hydrolysis. The molar absorptivity of $p$-nitrophenol is $18.290 \mathrm{M}^{-1} \mathrm{~cm}^{-1}$ and one unit of paraoxonase activity is equal to $1 \mathrm{~mol}$ of paraoxon hydrolyzed per liter per minute at $37^{\circ} \mathrm{C}$.

Phenylacetate was used as a substrate to measure the ARE activity. PON1, present in the sample, hydrolyses phenylacetate to its products, which are phenol and acetic acid. The produced phenol is colorimetrically measured via oxidative coupling with 4-aminoantipyrine and potassium ferricyanide. Nonenzymatic hydrolysis of phenyl acetate was subtracted from the total rate of hydrolysis. The molar absorptivity of colored complex is $4000 \mathrm{M}^{-1} \mathrm{~cm}^{-1}$ and one unit of arylesterase activity is equal to $1 \mathrm{mmol}$ of phenylacetate hydrolyzed per liter per minute at $37^{\circ} \mathrm{C}$.

Measurement of total free sulfhydryl groups of serum samples. Free sulfhydryl groups $(-\mathrm{SH})$ in serum samples were assayed according to the method of Ellman [21] as modifi ed by $\mathrm{Hu}$ et al. [22]. Briefl y, $1 \mathrm{~mL}$ of buffer containing 0.1 $\mathrm{M}$ Tris, $10 \mathrm{mM}$ EDTA (pH 8.2), and $50 \mu \mathrm{L}$ serum was added to cuvettes, followed by $50 \mu \mathrm{L}$ of $10 \mathrm{mM}$ DTNB in methanol. Blanks were run for each sample as a test, but without DTNB. Following incubation for $15 \mathrm{~min}$ at room temperature (RT), sample absorbance was read at $412 \mathrm{~nm}$ on a spectrophotometer . Sample and reagent blanks comprised the substrate. The concentration of sulfhydryl groups was calculated using reduced glutathione as the free sulfhydryl group standard, and the results are expressed as millimoles. The $\mathrm{CV}$ for the measurement of the serum - SH level was 3.6\%.

Measurement of the total antioxidant status of serum. The TAS of the serum was measured using a novel automated colorimetric measurement method for TAS developed by Erel [16]. In this method, antioxidants in the sample reduce dark blue-green colored 2, 2'-azino-bis (3-ethylbenzthiazoline-6sulphonic acid) (ABTS) radical to colorless reduced ABTS form. The change of absorbance at $660 \mathrm{~nm}$ is related with total antioxidant level of the sample. This method determines the antioxidative effect of the sample against the potent free radical reactions initiated by the produced hydroxyl radical. The results are expressed as micromolar trolox equivalent per liter.

Measurement of the total oxidant status of serum. The TOS of the plasma was measured using a novel automated colorimetric measurement method for TOS developed by Erel [15]. In this method, oxidants present in the sample oxidize the ferrous ion-chelator complex to ferric ion which makes a colored complex with a chromogen in an acidic medium. The color intensity, which can be measured spectrophotometrically, is related to the total amount of oxidant molecules present in the sample. The results are expressed in terms of micromolar hydrogen peroxide equivalent per liter ( $\mu \mathrm{mol}$ $\mathrm{H}_{2} \mathrm{O}_{2}$ Equiv. /L).

Oxidative stress index. The percentage ratio of TOS level to TAS level was accepted as oxidative stress index (OSI) [17]. For calculation, the resulting micromolar unit of TAS was changed to millimoles per liter, and the OSI value was calculated according to the following formula: OSI (arbitrary unit) $=$ TOS (micromolar hydrogen peroxide equivalent per liter)/TAS (micromolar trolox equivalent per liter).

Routine parameters. The levels of triglycerides (TG), total cholesterol (TC), HDL-cholesterol (HDL-C) and LDLcholesterol (LDL-C) were determined by using commercially available assay kits (Abbott) with an autoanalyzer (Architect - c16000, Abbott Diagnostics).

Statistical analysis. Statistical analyses were carried out using the statistical software version 11.5.1.0 (MedCalc, Mariakerke, Belgium). In normally distributed groups the results were presented with mean and SD, otherwise with medians. The significance of the differences between groups was determined by Student's unpaired t-test for normal distributions, and by the Mann-Whitney U-test in abnormal distributions. The Receiver Operating Characteristic (ROC) curve was used to identify the best diagnostic value as a MM predictor. $P$ values less than 0.05 was accepted as the significance level.

\section{Results}

Smokers were more common in CRC patients (35\%) than controls (20.5\%), but it did not show a significant difference $(p=0.23)$. Nineteen of CRC patients had a positive family history. When lipid parameters were compared, TC and HDL-C levels were significantly lower in the patients $(p=0.001$ and $\mathrm{p}<0.06$, respectively). Demographic and clinical data obtained from CRC patients and controls are summarized in Table 1.

Table 1. Clinical features of patients with CRC and controls were similar except the family history, TC of CRC in patients

\begin{tabular}{lllr}
\hline Parameter & Patients $(\mathbf{n}=\mathbf{4 0})$ & Controls $(\mathbf{n}=39)$ & $\boldsymbol{P}$ \\
\hline $\begin{array}{l}\text { Age, mean } \pm \text { SD } \\
\text { Gender }\end{array}$ & $56.5 \pm 2.1$ & $56.02 \pm 1.7$ & 0.24 \\
Male, $(\mathbf{n}),(\%)$ & & \\
Female, (n),(\%) & $21(\% 52.5)$ & $17(\% 43.6)$ & 0.57 \\
Smoker, (n),(\%) & $19(\% 47.5)$ & $22(\% 56.4)$ & 0.57 \\
BMI $\left(\mathbf{k g} / \mathbf{m}^{2}\right)$, mean \pm SD & $14(\% 35)$ & $8(\% 20.5)$ & 0.23 \\
Family history(n),(\%) & $25.74 \pm 2.54$ & $25.15 \pm 4.0$ & 0.43 \\
Lipid profile & $19(\% 47.5)$ & 0 & $0.001^{*}$ \\
TC, mean \pm SD (mg/dl) & $148.2 \pm 23.5$ & $167.9 \pm 26.2$ & $0.001^{*}$ \\
TG, mean \pm SD (mg/dl) & $125.6 \pm 28.5$ & $145.3 \pm 98$ & 0.44 \\
HDL, mean \pm SD (mg/dl) & $35.5 \pm 7.7$ & $38.62 \pm 5.2$ & 0.06 \\
LDL, mean \pm SD (mg/dl) & $104.5 \pm 9.7$ & $100.3 \pm 25.3$ & 0.43 \\
\hline
\end{tabular}

*statistically significant, TC:Total Cholesterol, TG:Triglycerid, LDL: Low Density Lipoprotein, HDL: High Density Lipoprotein. 
PON1 activities (PON and ARE) were significantly lower in patients with CRC compared to controls ( $p<0.001$ for each), whereas oxidant parameters (TOS and OSI) were significantly higher in CRC patients ( $p<0.001$ for each). However, antioxidant parameters (TAS and Ttl) significantly decreased in patients with CRC ( $p=0.03$ and $\mathrm{p}<0.0001$, respectively) (Table-2).

The final pathology reports of patients were evaluated, and subjects were divided into 3 groups according to the tumor grades. Statistically significant difference was obtained in Ttl, being highest in grade 1 followed by grade 2 and lowest in grade 3 tumors (Table 3 ).

Optimal cut-off levels and associated diagnostic performances (sensivity, specificity, and diagnostic value) of Ttl, PON, ARE, TAS, TOS, OSI based on ROC analysis, was given in Table 4 . The sensitivity and specificity of Ttl were $97.5 \%$ and 92.3\%, respectively, and the Area Under Curve (AUC) was

Table 3. When the cancer patients were subdivided into grades and compared, only Ttl was statistically different. Average rank for the abnormal distrubition

\begin{tabular}{lccccc}
\hline GRADE(n) & PON & ARE & Ttl & TAS & TOS \\
\hline $\mathbf{1}(6)$ & 14.5 & 23.75 & 23.17 & 14.75 & 23.58 \\
$\mathbf{2}(27)$ & 21.52 & 19.33 & 21.26 & 23.17 & 17.56 \\
$\mathbf{3}(7)$ & 21.71 & 22.21 & 15.29 & 15.14 & 29.21 \\
\hline $\mathbf{P}$ & 0.39 & 0.64 & 0.03 & 0.11 & 0.06 \\
\hline
\end{tabular}

Table 2. Serum PON1, ARE, TAS, TOS and OSI levels of CRC patients compared to the controls

\begin{tabular}{llll}
\hline Parameter $($ mean \pm SD $)$ & Patients $(\mathbf{n}=\mathbf{4 0})$ & Controls $(\mathbf{n}=\mathbf{3 9})$ & \multicolumn{1}{c}{$\boldsymbol{P}$} \\
\hline PON1 $(\mathbf{U} / \mathbf{L})$ & $128.2 \pm 63.2$ & $230.5 \pm 97.9$ & $<0.0001$ \\
Ttl $(\mathbf{m m})$ & $1427 \pm 233.6$ & $2064 \pm 200$ & $<0.0001$ \\
ARE $(\mathbf{k U} / \mathbf{L})$ & $150.6 \pm 49.2$ & $230.7 \pm 53.8$ & $<0.0001$ \\
TAS $(\mathbf{n m o l}$ Troloks/L) & $2.68 \pm 0.49$ & $2.87 \pm 0.28$ & 0.03 \\
TOS $\left(\mu\right.$ molH $_{2} \mathbf{O}_{2}$ Equiv./L) & $4.85(2.55-6.94)$ & $1.40(1.01-1.68)$ & $<0.0001$ \\
OSI & $0.13(0.10-0.27)$ & $0.05(0.04-0.06)$ & $<0.0001$ \\
\hline
\end{tabular}

* The result values; for the normal distrubition (mean \pm SD), for the abnormal distrubition (median)(IQR).
0.978. According to the ROC curve for ARE, the diagnostic sensitivity and specificity were $87.5 \%$ and $76.9 \%$, respectively, and the AUC was 0.862 (Figure 1). The AUC for the PON and OSI were $0.809,0.862$ respectively, and these values were lower than that of Ttl (0.978) and ARE.

\section{Discussion}

Data obtained in this study demonstrated a significant decrease in HDL-dependent PON activities (PON1 and ARE) and in Ttl levels in subjects with CRC compared with healthy subjects, whereas OSI and TOS levels were significantly increased. However, our results showed increased serum Ttl levels in patients with high grade CRC. This finding suggests that serum Ttl levels are important factors for the prognosis. To our knowledge, this is the first report demonstrating lower serum PON1, ARE activities in colorectal cancer patients. Results obtained here confirm that oxidative stress is involved in carcinogenesis when antioxidant defenses are unable to counteract free radicals.

Our findings may suggest that serum Ttl levels can be used as a novel serological biomarker in CRC patients. Currently, CEA is the most commonly used marker for CRC, however, this marker lacks the sensitivity and specificity needed to screen an average risk population [23]. Therefore, new biomarkers of cancer are needed that will further enhance detection of the disease and trigger a follow-up colonoscopy. It has been demonstrated that the 5-year survival period is increased when the disease is discovered in an early stage. It is, therefore, important to find new, reliable markers enabling an early diagnosis of this pathology.

Colon cancer is a major health problem, particularly because of the number of patients affected each year. Finding the biomarker or biomarkers with high sensitivity and specificity in colorectal cancer, and thus a high diagnostic value will determine their clinical usefulness in clinical practice. A comprehensive understanding of the relevance of each biomarker will be very important not only for diagnosing the disease reliably, but also help in the choice of multiple therapeutic alternatives currently available that is likely to benefit the patients(e.g,suplementattion of antioxidant) [24,25]. An increasing body of evidence suggests that paraoxonases are

Table 4. Optimal cut-off levels and associated diagnostic of PON, ARE, TAS, TOS, and OSI based on ROC analysis in CRC patients and confirmed Tt measurements to be superior to others

\begin{tabular}{|c|c|c|c|c|c|c|}
\hline Biomarker & Cut-off & Sensitivity(\%) & Specificity(\%) & $\mathrm{AUC}^{*}$ & $+\mathrm{LR}^{\#}$ & $-\mathrm{LR}^{*}$ \\
\hline T.Thiol & 1844 & 97.5 & 92.3 & 0.978 & 12.68 & 0.03 \\
\hline ARE & 209 & 87.5 & 76.9 & 0.862 & 3.79 & 0.16 \\
\hline TOS & 2.5 & 67.5 & 89.7 & 0.829 & 6.58 & 0.36 \\
\hline OSI & 0.09 & 70 & 89.7 & 0.825 & 6.83 & 0.33 \\
\hline PON1 & 209 & 90.2 & 64.1 & 0.809 & 2.51 & 0.15 \\
\hline TAS & 2.5 & 40 & 89.7 & 0.696 & 3.90 & 0.67 \\
\hline
\end{tabular}

${ }^{*}$ AUC: Area Under Curve ${ }^{\sharp}+$ and - Likelihood Ratio 


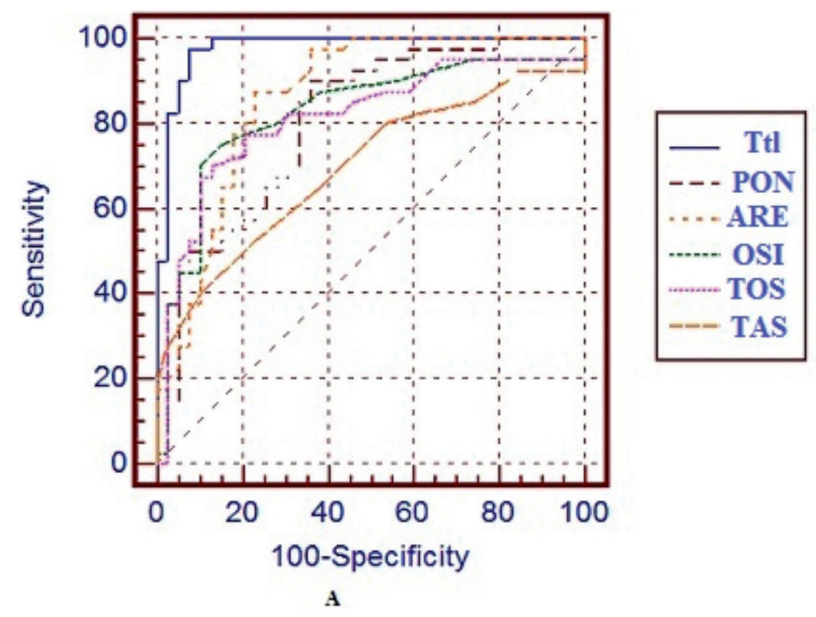

Ttl

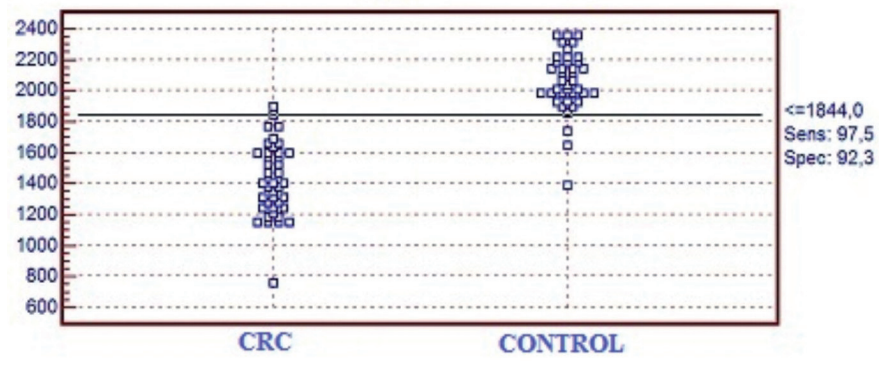

B

Figure 1. Diagnostic performances of Ttl, PON, ARE, OSI, TAS, and TOS in CRC patients, based on ROC analysis demonstrated ARE enzyme activity measurements to be a better predictor for HDL-dependent PON1 activity (A). Optimal cut-off level, sensitivity, specificity, and diagnostic value of Ttl (B).

associated with tumorogenesis. Recent studies revealed that PON2 and $P O N 3$, via interaction with coenzyme $Q_{10}$, diminish $\mathrm{O}_{2}{ }^{-}$release on either side of the inner mitochondrial membrane. This results in both lowered cardiolipin peroxidation and cytochrome $\mathrm{C}$ release, providing a marked resistance against apoptosis [11]. In the absence of an acknowledged biological substrate for PON1, its activity is measured through its degrading function towards the artificial substrates paraoxon and phenyl acetate. Recent studies indicate that arylesterase activity best reflects the antioxidant activity of PON1, although it is not directly responsible for it [3]. There is evidence in some studies that PON1's antioxidant function begins at the level of lipoprotein (LDL and HDL) protection against oxidative modification by ROS. The enzyme also reduces lipid hydroperoxides to hydroxides and presents a peroxidase- like activity, as PON1 was shown to degrade hydrogen peroxide $\left(\mathrm{H}_{2} \mathrm{O}_{2}\right)$, a major reactive oxygen species produced under oxidative stress. The increased production of mitochondrial ROS can be induced, for example, by oxidized LDL. So mitochondrial dysfunction leads to decreases in aerobic capacity, which is a strong predictor of mortality. Increased production of mitochondrial ROS also causes endothelial dysfunction/apoptosis, which finally leads to the development of cancer.

Plasma thiol groups are critical endogenous antioxidants that act concurrently in scavenging and/or reducing free radicals, thus breaking the peroxidative chain and allowing the repair of oxidatively damaged molecules. Thus, the reduction of total thiol groups observed newly diagnosed unterated patients confirms a mechanism of action of these patients that might evoke an adaptive response resulting in an increased TOS. Notably, the - SH groups of proteins are an important component of the antioxidant mechanism. The decreased PON1 activity during oxidative stress has been primarily attributed to changes in the redox status of the free sulfhydryl groups, since sulfhydryl compounds prevented the inhibition of PON1 activity caused by ROS [26,27]. However, Serum Ttl levels in patients with high grade CRC are a direct measure of the in vivo redox status in humans, because thiols react readily with oxygen-containing free radicals to form disulfides. Serum thiols also reflect DNA repair capacity and the possible eventual accumulation of genetic damage, since a key DNA repair enzyme, poly ADP-ribose polymerase, is thiol/disulfide redox regulated [28]. Therefore Ttl levels may serve as additive non-invasive biochemical markers of oxidative stress in colon cancer and this hypothesis needs to be further investigated.

Because the antioxidant status of human plasma is dynamic and can be affected by various factors, including diet, physical exercise, injury, and disease, the relationship between TOS of serum and OSI, TAS and total thiol groups may better reflects real oxidative stress and health status $[29,30]$.

One major limitation of the study is the small number of samples. To validate a more accurate diagnostic value of Ttl and ARE/PON, a large scale study including cancer/inflammatory disease should be performed in the future. Antioxidant Ttl and free radical scavenger (PON 1) enzymes reduce oxidative stress and thus act as central regulators of diseases, including CRC.

Other evidence emerging from these data suggest that, in addition to their positive effect on general health, antioxidants may also exert specific beneficial effects on tumor progression and may represent a valid therapeutic support during treatment.

\section{References}

[1] WITTE I, FOERSTERMANN U, DEVARAJAN A, REDDY ST, HORKE S. Protectors or Traitors: The Roles of PON2 and PON3 in Atherosclerosis and Cancer. J Lipids. 2012. http:// dx.doi.org/10.1155/2012/342806

[2] LOSCALZO J. Paraoxonase and coronary heart disease risk: language misleads, linkage misinforms, function clarifies. Circ 
Cardiovasc Genet. 2008; 1: 79-80. http://dx.doi.org/10.1161/ CIRCGENETICS.108.837179

[3] YILMAZ N. Relationship between paraoxonase and homocysteine: crossroads of oxidative diseases.Arch Med Sci. 2012 29; 8: 138-53.

[4] DRAGANOV D. Human paraoxonases (PON1, PON2, and PON3) are lactonases with overlapping and distinct substrate specificities J Lipid Res 2005; 46: 1239-47. http://dx.doi. org/10.1194/jlr.M400511-JLR200

[5] YILMAZ N, AYDIN O, YEGIN A, TILTAK A, EREN E et al. Impaired oxidative balance and association of blood glucose, insulin and HOMA-IR index in migraine. Biochem Med (Zagreb). 2011; 21: 145-51. http://dx.doi.org/10.11613/BM.2011.023

[6] LEUFKENS AM, VAN DUIJNHOVEN FJ, WOUDT SH, SIERSEMA PD, JENAB M, et al. Biomarkers of oxidative stress and risk of developing colorectal cancer: a cohort-nested casecontrol study in the European Prospective Investigation Into Cancer and Nutrition. Am J Epidemiol. 2012; 1: 175:653-63

[7] YILMAZ N, AYDIN O, YEGIN A, TILTAK A, EREN E. Increased levels of total oxidant status and decreased activity of arylesterase in migraineurs. Clin Biochem. 2011; 44(10-11): 832-7. http://dx.doi.org/10.1016/j.clinbiochem.2011.04.015

[8] BOBIN-DUBIGEON C, JAFFRE I, JOALLAND MP, CLASSE JM, CAMPONE M, et al. Paraoxonase 1 (PON1) as a marker of short term death in breast cancer recurrence. Clin Biochem. 2012 [Epub ahead of print]. http://dx.doi.org/10.1016/j.clinbi ochem.2012.05.021

[9] BALCI H, GENC H, PAPILA C, CAN G, PAPILA B et al. Serum lipid hydroperoxide levels and paraoxonase activity in patients with lung, breast, and colorectal cancer. J Clin Lab Anal. 2012; 26: 155-60. http://dx.doi.org/10.1002/jcla.21503

[10] ARIOZ DT, CAMUZCUOGLU H, TOY H, KURT S, CELIK $\mathrm{H}$ et al. Assessment of serum paraoxonase and arylesterase activity in patients with endometrial cancer. Eur J Gynaecol Oncol. 2009; 30: 679-82.

[11] ROTHEM L, HARTMAN C, DAHAN A, LACHTER J, ELIAKIM R, et al. Paraoxonases are associated with intestinal inflammatory diseases and intracellularly localized to the endoplasmic reticulum. Free Radic Biol Med. 2007; 43: 730-9. http://dx.doi.org/10.1016/j.freeradbiomed.2007.05.003

[12] MACKNESS B, BELTRAN-DEBON R, ARAGONES G, JOVEN J, CAMPS J, et al. Human tissue distribution of paraoxonases 1 and 2 mRNA. IUBMB Life. 2010; 62: 480-2.

[13] RODRIGO L, HERNANDEZ AF, LOPEZ-CABALLERO JJ, GIL F, PLA A. Immunohistochemical evidence for the expression and induction of paraoxonase in rat liver, kidney, lung and brain tissue. Implications for its physiological role. Chem Biol Interact. 2001; 137: 123-37. http://dx.doi.org/10.1016/S00092797(01)00225-3

[14] ROMANI R, DE MEDIO GE, DI TUllio S, LAPALOMBELLA R, PIRISINU I, et.al. Modulation of paraoxonase 1 and 3 expression after moderate exercise training in the rat. J Lipid Res. 2009; 50: 2036-45. http://dx.doi.org/10.1194/jlr. M800493-JLR200

[15] EREL O. A new automated colorimetric method for measuring total oxidant status. Clin Biochem 2005; 38: 1103-11. http://dx.doi.org/10.1016/j.clinbiochem.2005.08.008
[16] EREL O. A novel automated direct measurement method for total antioxidant capacity using a new generationmore stable ABTS radical cation. Clin Biochem 2004; 37: 277-85. http://dx.doi.org/10.1016/j.clinbiochem.2003.11.015

[17] HARMAM, HARMA M, EREL O. Increased oxidative stress in patients with hydatidiform mole. Swiss MedWkly 2003; 133: 563-6.

[18] CHUNG KY, LEE SJ, CHUNG SM, LEE MY, BAE ON, et al. Generation of free radical by interaction of iron with thiols in human plasma and its possible significance. Thromb Res 2005; 116: 157-64. http://dx.doi.org/10.1016/ j.thromres.2004.11.021

[19] SEN CK. Redox signaling and the emerging therapeutic potential of thiol antioxidants. Biochem Pharmacol 1998; 55: 1747-58. http://dx.doi.org/10.1016/S0006-2952(97)00672-2

[20] WLODEK L. Beneficial and harmful effects of thiols. Pol J Pharmacol 2002; 54: 215-23.

[21] CAROLINA M, DA COSTA, RITA CC DOS SANTOS, EMERSON SL. A simple automated procedure for thiol measurement in human serum samples J Bras Patol Med Lab 2006; 42: 345-50.

[22] HU ML. Measurement of protein thiol groups and glutathione in plasma. Methods Enzymol. 1994; 233: 380-5. http://dx.doi. org/10.1016/S0076-6879(94)33044-1

[23] LEE H, SONG M, SHIN N, SHIN CH, MIN BS, et al. Diagnostic significance of serum HMGB1 in colorectal carcinomas. PLoS One. 2012; 7: 34318. http://dx.doi.org/10.1371/journal. pone.0034318

[24] ANANT NB, ROHIT M, ABDULLAH F, AMIT V, DWARAKANATH BS. Cancer biomarkers - Current perspectives. Indian J Med Res 2010; 132: 129-49

[25] BART $Ł K$, TADEUSZ $€$. The evaluation of diagnostic value of the tumor markers: CCSA-2 and CEA in colorectal cancer. Polski Przegląd Chirurgiczny 2012; 84: 86-92.

[26] ROZENBERG O, AVIRAM M. S-Glutathionylation regulates HDL-associated paraoxonase 1 (PON1) activity. Biochem Biophys Res Commun 2006; 351: 492 - 8. http://dx.doi. org/10.1016/j.bbrc.2006.10.059

[27] ERTURK C, ALTAY MA, SELEK S, KOCYIGIT A. Paraoxonase-1 activity and oxidative status in patients with knee osteoarthritis and their relationship with radiologicaland clinical parameters. Scand J Clin Lab Invest. 2012 [Epub ahead of print]. http://dx.doi.org/10.3109/ $\underline{00365513.2012 .687116}$

[28] ARTHUR F BANNE, AMIR AMIRI, RONALD W PERO. Reduced level of serum thiols in patients with a diagnosis of active disease. J Anti Aging Med. 2003; 6(4): 327-34. http:// dx.doi.org/10.1089/109454503323028920

[29] ICIEK M, CHWATKO G, LORENC-KOCI E, BALD E, WLODEK L. Plasma levels of total, free and protein bound thiols as well as sulfane sulfur in different age groups of rats. Acta Biochim Pol. 2004; 51: 815-24.

[30] GIUSTARINI D, ROSSI R, MILZANI A, COLOMBO R, DALLE-DONNE I. S-Glutathionylation: from redox regulation of protein functions to human diseases. J Cell Mol Med 2004; 8: 201-12. http://dx.doi.org/10.1111/j.1582-4934.2004. $\underline{\mathrm{tb} 00275 . \mathrm{x}}$ 